\title{
RELEVANCIA DE LA INTERVENCIÓN EDUCATIVA COMO FACTOR PREVENTIVO DE LA ANEMIA EN NIÑOS ENTRE 6 Y 36 MESES: EL CASO DEL ÁREA URBANO MARGINAL DE FLOR DE AMANCAES, LIMA, PERÚ
}

\author{
IMPORTANCE OF EDUCATIONAL INTERVENTION AS A PREVENTIVE \\ FACTOR OF ANEMIA IN CHILDREN BETWEEN 6 AND 36 MONTHS OLD: \\ THE CASE OF INNER-CITY AREAS OF FLOR DE AMANCAES, LIMA, PERU
}

\author{
Iván Hidalgo Romero \\ Ingeniero Economista \\ Universidad Científica del Sur \\ ihidalhor@usmp.pe \\ Perú, Lima
}

\section{SUMARIO}

- Introducción.

- Antecedentes.

- Sobre la anemia infantil en el Perú.

- Experiencia e Intervención Educativa.

- Conclusiones.

\section{RESUMEN}

En el contexto del derecho a la vida $-y$ particularmente a la vida de los niños y su bienestar integral- la legislación nacional e internacional consagran este derecho. Al ser la anemia uno de los factores que más influye tanto en la morbilidad infantil, así como en el desarrollo de sus facultades, es indispensable validar estrategias conducentes a su disminución/eliminación.

La investigación se sustentó en el marco conceptual del Plan Nacional para la Reducción y Control de la Anemia Materno Infantil en el Perú, 2017-2021. En este sentido y a partir de la opinión de expertos, médicos, nutricionistas, madres líderes, entre otros, la propuesta fue demostrar el alcance de una intervención educativa que permitiera la reducción de la anemia en niños entre 6 y menos de 36 meses de edad entre octubre 2018 y febrero 2019 en el asentamiento humano Flor de Amancaes. Se concluye que el control y efecto de la anemia en áreas urbano marginales en el Perú dependerá del nivel de educación, de la capacitación con acompañamiento de los padres de familia y del compromiso legal del Estado en velar por el derecho a la vida a través del cuidado nutricional de nuestros niños.

\begin{abstract}
In the context of the right to life -and particularly to the life of children and their fundamental well-being- the national and international legislation enshrines this right. Since anemia is one of the factors that most influences both infant morbidity and the development of their faculties, it is essential to validate strategies that lead to its reduction / elimination.
\end{abstract}

The research was founded on the conceptual framework of the National Plan for the Reduction and Control of Childhood and Maternal Anemia in Peru, 2017-2021. In this senseand from the opinion of experts, doctors, nutritionists, leading mothers, among others, the proposal was to demonstrate the scope of an educational intervention that allows the reduction of anemia in children between 6 and less than 36 months old from October 2018 and February 2019 in Flor de Amancaes shanty town. It is concluded that the control and effect of anemia in inner city areas in Peru will depend on the level of education, training with accompaniment of parents and the legal commitment of the State to ensure the right to life through the nutritional care of our children. 


\section{PALABRAS CLAVE}

Anemia, hierro, micronutrientes, intervención educativa, programa de gobierno, salud pública.

\section{KEYWORDS}

Anemia, iron, micronutrients, educational intervention, government program, public health.

\section{INTRODUCCIÓN}

El sistema internacional mediante tratados en derechos humanos, ha ido generando una serie de obligaciones que los estados deben satisfacer teniendo como eje central de sus acciones la visión de la persona o del ciudadano, es lo que se ha venido en llamar desarrollo de políticas públicas con enfoque de derechos humanos, que implica hacer efectivos los compromisos adquiridos, a nivel nacional teniendo como norte que los derechos fundamentales como vida, salud, integridad y educación -entre otros-, no sólo son exigibles en el papel, sino que constituye obligación estatal hacer realidad su disfrute proveyendo el marco legal y las condiciones políticas e institucionales para lograr un real disfrute.

El tener esta visión de enfoque de derechos humanos en el quehacer cotidiano de la gestión y programación de políticas públicas, a ser ejecutadas por las diferentes instancias gubernamentales, implica dejar de lado la clásica visión asistencialista de apoyo a la población, para asumir la intervención estatal como un mecanismo fundamental que, busque el acceso a los ciudadanos a gozar en forma concreta de derechos, lo que implica proveer medidas concretas y medibles.

Ahora para poder comprender el contexto de la importancia de abordar este tema, hay que tener claro que desde el siglo $\mathrm{XX}$, cuando se aprobó la Declaración Universal de los Derechos Humanos, se estableció en su artículo 25 , que toda persona cuenta con el derecho de asistencia médica (Asamblea General de las Naciones Unidas 1948) y, es dicha regulación la piedra angular para el desarrollo de los planes de salud y leyes de los países, entre los que se encuentra el Perú.

Esta línea de pensamiento, se desprende al acuerdo adoptado por la organización de las
Naciones Unidas en el septuagésimo periodo de sesiones celebrado en New York en setiembre del 2015, en el cual se aprobaron los 17 Objetivos de Desarrollo Sostenible ODS, en los considerandos que sustentan la declaración se tiene en mente lograr que en 15 años se logre erradicar la pobreza y permitir el desarrollo en tres aspectos el social, económico y ambiental (Naciones Unidas 2015). Para lo cual cada Estado debe desarrollar en forma objetiva una agenda que permitan asegurar el pleno acceso a los derechos de las personas con la implementación de medidas concretas que le mejoren la vida.

La política pública de lucha concreta contra la anemia, que viene implementando el Estado peruano, se encaja dentro de los ODS siguientes: fin de la pobreza, salud y bienestar, educación de calidad y reducción de la desigualdad, entre otros.

También es preciso tener en consideración que, la salud es lo que se denomina un derecho puerta, es decir que va a permitir a la persona, poder acceder a otros derechos fundamentales, como son la educación y el pleno desarrollo del individuo. Es decir, que sin salud no podemos hablar de un individuo con goce de derechos.

En el documento Salud y Derechos Humanos de la Organización Mundial de la Salud, vamos a encontrar que los Estados cuando desarrollen programas de atención en salud, tienen que promover los derechos fundamentales de las personas, en cumplimiento de sus obligaciones teniendo presente los criterios siguientes (Organización Mundial de la Salud 2017): (a) No discriminación. (b) Disponibilidad. (c) Accesibilidad. (d) Aceptabilidad. (e). Calidad. (f) Rendición de cuentas. (g) Universalidad.

Por otro, lado en el Perú tenemos la ley $\mathrm{N}^{\circ}$ 26842 (Ley General de la Salud), cuyo artículo segundo del título preliminar, señala que: "la protección de la salud es de interés público. Por tanto, es responsabilidad del Estado regularla, vigilarla y promoverla" (Congreso de la República 1997, ley N² 26842, Artículo 2 del título preliminar).

Expuesto el marco normativo mundial y nacional que obliga al Estado peruano, pasemos a analizar los otros aspectos del contexto de la realidad que convoca la presente investigación. 
Así mismo, los diversos factores causales de la anemia aquejan a más de 293 millones de niños del planeta, donde el $47 \%$ es debido a la deficiencia de hierro -anemia ferropénica o ferropriva-(Balajaran, Ramakrishnan, Ozaltin, Shankar y Subramanian 2011). Existen otras causas, como la carencia de folato, vitamina B12, vitamina A, entre otras, que contribuyen también a la prevalencia de la anemia.

Lamentablemente la anemia es un problema de salud pública a nivel mundial. (Kibret, Chojenta, D'Arcy, \& Loxton 2019). La investigación científica demuestra que aparte de la morbilidad prematura- una de las principales consecuencias que desencadena la anemia, es la afección al desarrollo psicomotor del niño y a su rendimiento escolar; la reducción de la capacidad intelectual, capacidad física y de trabajo.

Otros estudios indican el $50 \%$ de las causales de la anemia se debe a la deficiencia de hierro, pero sin haber considerado las grandes brechas sociales y económicas entre regiones. (Gonzales; Huamán-Espino; Gutiérrez; Aparco y Pillaca 2015); sin embargo, Zabaleta \& Astete (2017), subrayan la importancia de "fortalecer las acciones para alcanzar una mayor adherencia y efectividad de la suplementación con hierro a la madre y al niño" (página 720). Según la encuesta realizada a las madres de informantes de esta investigación, se determina una clara relación entre el poco conocimiento sobre qué es la anemia, sus causas y sus consecuencias y su vínculo con la concientización de la importancia de tomar los multimicronutrientes para la prevención de la anemia (Junco, 2015).

Huamán-Espino; Aparco; Núñez-Robles; Gonzales; Pillaca \& Mayta-Tristán (2012) encontraron que no solo es consumir la cantidad requerida de multimicronutrientes sino que es necesario que la manera de preparación y del consumo deben ser los adecuados para la correcta asimilación. Sin embargo, según Aparco; Bullón \& Cusirramos (2019), discrepan con Huamán-Espino, et al. (2012) indicando que, su diseño de estudio no consideró a niños que consumieron multimicronutrientes y los que no consumieron la cantidad necesaria de multimicronutrientes.
Respecto al modo de enfrentar el problema, es fundamental trabajar el tema con organizaciones sociales y padres de familia para sensibilizar y concientizar a la población sobre la importancia de prevenir y tratar la anemia. Aunque, el descarte preventivo de la anemia a temprana edad es clave, es indispensable realizar diversas campañas que permitan informar a las madres de los peligros y riesgos que acarrea no tratar a tiempo la anemia por factores de nutrición durante los primeros 36 meses de vida del niño. (Arroyo-Laguna, 2017). Y es aquí donde cobra relevancia el hecho de que las estrategias de prevención de la anemia deban centrarse, en mujeres con limitaciones educativas en zonas rurales con carente disponibilidad de recursos básicos para la higiene y saneamiento tanto de la madre como del niño, en estrategias de prevención que supongan una intervención educativa integral.

En ese sentido, es fundamental trabajar el tema con organizaciones sociales y padres de familia para prevenir y tratar la anemia a tiempo.

Analizando la información del cuadro $\mathrm{N}^{\circ} 1$ se establece la importancia de otras investigaciones que definen a la anemia a un nivel de hemoglobina bajo $(<110 \mathrm{~g} / \mathrm{L}$ para mujeres embarazadas $\mathrm{y}<120 \mathrm{~g} / \mathrm{L}$ para mujeres no embarazadas). Si el nivel de hemoglobina de un individuo es bajo, los glóbulos rojos no pueden transportar el oxígeno adecuado para las necesidades fisiológicas del cuerpo.

En consecuencia, las intervenciones más eficientes, son aquellas que tienen un enfoque de ciclo de vida que incorpora la educación, prevención y la recuperación de la salud, otros factores de anemia, son la deficiencia de folato, vitamina $\mathrm{B} 12$, vitamina $\mathrm{A}$; infecciones agudas, la parasitosis, la intoxicación por metales pesados y condiciones genéticas o adquiridas que afectan, así pues, la síntesis de hemoglobina y la producción o multiplicación de los glóbulos rojos. Todo ello refuerza como lo adelantamos- el requerimiento de una intervención educativa integral. 


\section{CUADRO $\mathrm{N}^{\circ} 1$. Valores normales de concentración de hemoglobina.}

\begin{tabular}{|c|c|c|c|c|}
\hline Población & \multicolumn{3}{|c|}{$\begin{array}{c}\text { Con anemia } \\
\text { Según niveles de hemoglobina (g/dL) }\end{array}$} & $\begin{array}{l}\text { Sin anemia } \\
\text { Si la concentración de } \\
\text { hemoglobina }(\mathrm{g} / \mathrm{dL})\end{array}$ \\
\hline \multicolumn{5}{|l|}{ Niños } \\
\hline \multicolumn{5}{|l|}{ Niños prematuros } \\
\hline $1^{a}$ semana de vida & \multicolumn{3}{|c|}{$\leq 13.0$} & $>13.0$ \\
\hline $2^{\mathrm{a}}$ a 4 ta semana de vida & \multicolumn{3}{|c|}{$\leq 10.0$} & $>10.0$ \\
\hline $5^{\mathrm{a}}$ a $8 \mathrm{va}$ semana de vida & \multicolumn{3}{|c|}{$\leq 8.0$} & $>8.0$ \\
\hline \multicolumn{5}{|l|}{ Niños nacidos a término } \\
\hline Menor de 2 meses & \multicolumn{3}{|c|}{$<13.5$} & $13.5-18.5$ \\
\hline \multirow[t]{2}{*}{ Niños de 2 a 6 meses cumplidos } & \multicolumn{3}{|c|}{$<9.5$} & $9.5-13.5$ \\
\hline & Severa & Moderada & Leve & \\
\hline Niños de 6 meses a 5 años cumplidos & $<7.0$ & $7.0-9.9$ & $10.0-10.9$ & $\geq 11.0$ \\
\hline Niños de 5 a 11 años de edad & $<8.0$ & $8.0-10.9$ & $11.0-11.4$ & $\geq 11.5$ \\
\hline
\end{tabular}

Fuente y elaboración: Ministerio de Salud del Perú

Es importante destacar que en los últimos ocho años la cantidad de infantes entre seis a 36 meses que tenían anemia ha mostrado un comportamiento estacionario y en algunos casos incluso superior al $41.6 \%$ del año 2011 , revirtiendo una tendencia sostenidamente declinante que se registraba desde el año 2007.

Entre las causas de lo estacionario y de la declinación es el consumo inadecuado de hierro y de otros micronutrientes a partir de los alimentos, como vimos la escasez de hierro y vitaminas acarrea como consecuencia la inapropiada formación de la hemoglobina.

Es a partir de este estancamiento/declinación en la reducción de la anemia en el Perú por espacio de más de cinco años, que el Instituto de Gobierno y Gestión Pública de la USMP -como parte de su programa de proyección social- ha considerado pertinente investigar para ampliar los conocimientos y estrategias que permitan plantear soluciones al problema de la anemia por deficiencia de hierro. Para ello se realizó una intervención educativa integral que permitiese su diagnóstico y prevención en niños/as entre seis a 36 meses de edad en el asentamiento humano Flor de Amancaes. Para ello se tomó como base el marco conceptual del Plan Nacional para la Reducción y Control de la Anemia Materno Infantil en el Perú: 2017 - 2021 del Ministerio de Salud -Minsa, además de la revisión de fuentes bibliográficas internacionales y nacionales.

\section{ANTECEDENTES}

La anemia como problema mundial de la salud, presenta factores causantes multifactoriales, coexistiendo simultáneamente. Sin embargo, el fundamental factor es la pobre ingesta alimenticia, que a pesar de diversos programas sociales estos no generan impactos positivos. Ello se manifiesta también en algunos lugares de Latinoamérica de manera crónica, debido a la baja lactancia materna, escasa ingesta de frutas $y$ vegetales, coadyuvado con enfermedades oportunistas como infecciones intestinales. (Pita-Rodríguez y JiménezAcosta, 2011) De igual modo, se vienen presentando muchos hallazgos de la anemia falciforme, cuya característica es de naturaleza genética con presencia de la hemoglobina o la llamada anemia de Fanconi, asociada además con mutaciones que originan cromosomas 
inestables en el individuo, fundamentalmente en la médula roja de los huesos (HernándezMartínez, 2018).

Asimismo, otra causa de la anemia, es la inflamación producida por infecciones crónicas o tumores que generan que el hierro no sea captado por la hemoglobina, sino más bien sea absorbido por el intestino, cuyo tratamiento requiere un control de la absorción intestinal (Feldman, Najle, Rivero, Rodríguez, Estein, 2017).

Ahora bien, para ejecutar sus acciones concretas en la promoción y defensa de los derechos fundamentales, el Estado peruano aprobó el Plan Nacional de Derechos Humanos 20182021. A efectos de tener una mejor supervisión de las acciones que plantea, se realiza un análisis etario de la población y se indica adicionalmente que entidad es la responsable de ejecutar las medidas que permitan alcanzar los objetivos (Ministerio de Justicia y Derechos Humanos s/f).

Así en dicho instrumento legal, se establece en el primer objetivo estratégico, "lograr el desarrollo de los niños, niñas y adolescentes en entornos seguros y, libres de violencia" (Ministerio de Justicia s/f, página 96); a través de una acción estratégica para afrontarla en niños que van de los 6 a 36 meses, señalando al Ministerio de Salud y al Ministerio de Inclusión Social, como los responsables de la ejecución, siendo la meta lograr una reducción de la anemia, para lo cual se establece al 2018 de $33.2 \%$, disminuya al $19 \%$ en el año 2021 .

Como podemos apreciar la política pública fundamental del Estado peruano, se focaliza en menores hasta los 36 meses, olvidando el resto del grupo de niños, niñas y adolescentes, que también padecen este flagelo, por lo cual este trabajo permitirá plantear un sistema de acción estratégica a menores en edad escolar, donde la anemia también está presente.

\section{SOBRE LA ANEMIA INFANTIL EN EL PERÚ}

El gobierno peruano, en los últimos años viene desarrollando normas que permiten una aplicación uniforme de los criterios para ejecutar una política pública de desarrollo infantil, lo cual se va a reflejar en la dación de una serie de pautas para lograr la articulación intersectorial e intergubernamental de intervenciones efectivas (Plan Multisectorial de Lucha contra la Anemia), aspirando a un control verdadero sobre un problema tan serio como es el de la anemia en niños y adolescentes. Precisamente ello ha permitido establecer como una de las causas de la anemia infantil, la situación sociocultural de la madre y su pobre conocimiento respecto al tema. Por ello el Ministerio de Salud, en el año 2017 dicta una Norma Técnica que dispone que se debe brindar en forma gratuita a las gestantes hierro y ácido fólico; ello unido a la obligatoriedad de llevar a cabo la detección de la situación de anemia en niños y adolescentes en los centros de atención o centros de salud. Es en ese contexto es importante determinar si las estrategias para la ejecución de la política pública son o no, apropiadas para afrontar el reto.

\section{Situación de la anemia infantil en el Perú}

El gráfico № 1 , muestra los casos de la anemia en el periodo del 2015 al 2017. Se aprecia que la reducción de la anemia en niños menores de 36 meses, no ha sido significativa, durante el periodo 2015 (43.5\%) al 2017 (43.6\%).

Esto demuestra la poca efectividad del actual gobierno frente al recalcitrante problema de la anemia aun cuando nuestro Producto Bruto Interno (PBI) en aspectos económicos haya sido estable comparado con otras realidades como la de Ecuador, Colombia y México, donde el nivel de anemia es menor al nuestro. Inclusive otros estudios de la anemia en la zona rural $(53.3 \%)$ pone en evidencia que esta es mayor en comparación con la urbana (40,0\%). (INEI, 2017, p.24). 


\section{GRÁFICO N$^{\circ}$ 1. Porcentaje de anemia en niños/as de 6 a 35 meses}

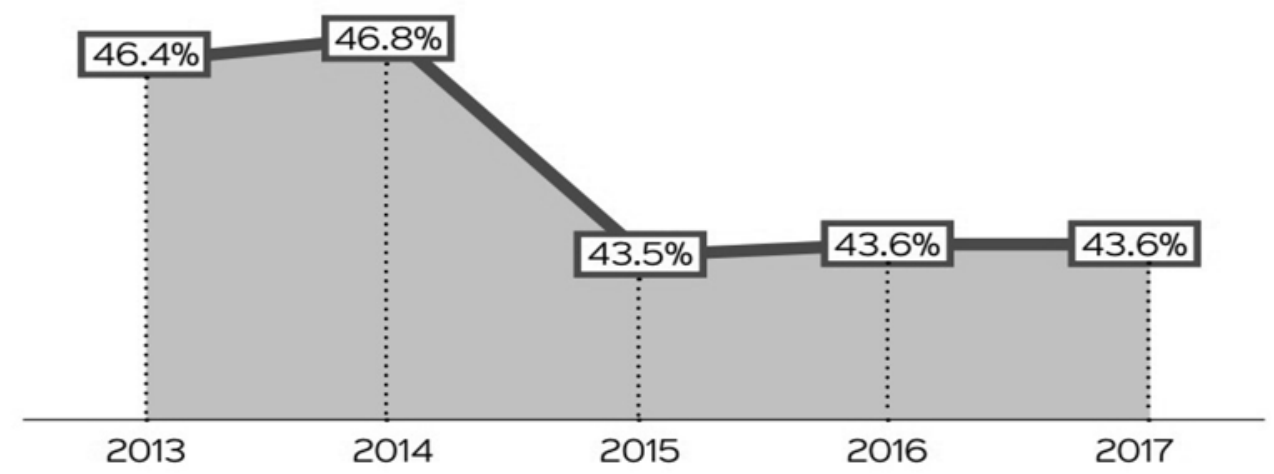

Fuente: Encuesta Demográfica y de Salud Familiar Endes-inei año 2017

Elaboración: Defensoría del Pueblo

Es importante mencionar que, si bien las acciones desarrolladas por el MINSA (2017) durante el periodo 2017 a través de la Norma Técnica vigente de salud para el control del crecimiento y desarrollo de los niños y niñas menores de cinco años privilegian las acciones de prevención y tratamiento de la anemia, los resultados preliminares no son alentadores pues evidencian que un $46.6 \%$ menores de 36 meses padecen anemia.

\section{La anemia como amenaza del derecho a la salud}

En ese contexto, los resultados obtenidos en el informe de la Defensoría del Pueblo, sobre la lucha contra la anemia resulta vital, pues nos permite evaluar la actividad integral que realiza el Estado, donde los planes sectoriales de lucha contra la anemia del MINSA y del MIDIS, no han cumplido con su objetivo de reducir la anemia infantil, es decir, hay que realizar intervenciones con el modelo que demuestre mejor eficacia en su ejecución.

En el aspecto de regulación normativa, vamos a encontrar que el Estado peruano se ha encargado de dictar las medidas necesarias para poder contar con una política pública apropiada para combatir los elevados índices de anemia que nuestra población de niños y adolescentes afronta; sin embargo, analizando los resultados, -en especial los de la Defensoría del Pueblo-, queda claro que no hay un acompañamiento a las madres de los niños, ni tampoco una articulación entre los sectores gubernamentales comprometidos. Por ello, el modelo que se presenta, al realizar el seguimiento concreto de la aplicación de los insumos requeridos por los menores para luchar contra la anemia, demuestra que tal actividad, es fundamental para poder lograr resultados efectivos en esta lucha.

\section{La intervención del Estado para la reducción de la anemia infantil.}

El gobierno del Estado peruano, en los últimos años viene desarrollando normas que permitan una aplicación uniforme de los criterios para ejecutar una política pública de desarrollo infantil, lo cual se va a reflejar en la dación de una serie de pautas para lograr la articulación intersectorial e intergubernamental de intervenciones efectivas (Ministerio de Desarrollo e Inclusión Social-2018), que permita un control verdadero sobre un problema tan serio como es el de la anemia en niños y adolescentes.

Habiéndose establecido que una de las causas de la anemia infantil, se sustenta en la situación similar de la madre, el Ministerio de Salud al dictar su Norma Técnica dispone que se debe brindar en forma gratuita a las gestantes hierro y ácido fólico; ello unido a la obligatoriedad de llevar a cabo la detección de la situación de anemia en niños y adolescentes en los centros de atención del Primer Nivel de Atención (Ministerio de Salud 2015); es en ese contexto 
donde va a resultar importante determinar si las estrategias para la ejecución de la política pública son apropiadas para afrontar el reto. En dicho contexto, el Informe de la Defensoría del Pueblo (2019) sobre los resultados en la lucha contra la anemia, resulta vital pues nos permite conocer la evaluación que realizan a la actividad integral del Estado que, los planes sectoriales de lucha contra la anemia del MINSA y del MIDIS, no han cumplido con su objetivo de reducir la anemia infantil, es decir hay que pasar a realizar intervenciones como las que el modelo mostrado ejecuta con eficacia.

En el aspecto de regulación normativa, vamos a encontrar que el gobierno del Estado peruano se ha encargado de dictar las medidas necesarias para poder contar con una política pública apropiada para combatir los elevados índices de anemia que nuestra población de niños y adolescentes afronta; sin embargo, analizando los resultados -en especial los de la Defensoría del Pueblo-, queda claro que no hay un acompañamiento a las madres de los niños, ni tampoco una articulación entre los sectores gubernamentales comprometidos.

Por lo tanto, los diferentes sectores de gobierno -como lo señala la Defensoría del Pueblo-, carecen de línea de base de intervención, no practican la ejecución de tareas conjuntas $\mathrm{y}$, lo más lamentable que se deje de ejecutar los fondos asignados para la lucha contra la anemia y, un dato esencial que el $59 \%$ de las familias entrevistadas, carece de una clara conciencia de las implicancias de la presencia de la anemia en el desarrollo de los niños y adolescentes.

Por ello el modelo que se presenta, al realizar el seguimiento concreto de la aplicación de las medicinas a los menores, demuestra que tal actividad, es fundamental para poder lograr resultados efectivos en la lucha contra la anemia.

\section{EXPERIENCIA E INTERVENCIÓN EDUCATIVA}

\section{Diseño del modelo de intervención educativa}

El enfoque metodológico consistió en transmitir conocimientos teórico-prácticos sobre la anemia en las madres cuidadoras, teniendo como punto de partida los saberes previos con los que cuentan las madres, desde su propia experiencia de vida, sus costumbres y su hábitat, que es necesario rescatar, valorar y en la medida de lo posible mejorar y/o complementar con la información brindada desde el conocimiento del campo nutricional, mejorando los hábitos en el cuidado y alimentación en los niños y niñas. El diseño comprendió tres pilares importantes: el equipo técnico de educación nutricional, las promotoras comunales y las madres cuidadores/as. El equipo técnico de educación nutricional, eje principal para la puesta en marcha del modelo de intervención educativa, tuvo como función vital el desarrollo de capacidades en las Promotoras Comunales, a través de capacitaciones dirigidas a ellas. Asimismo, el equipo técnico - como otra de sus funciones- monitoreó y validó los mensajes en hogares de niños y niñas con desnutrición. Cumpliendo, asimismo, con retroalimentar la intervención educativa, en base a lo recogido de las experiencias de campo y evaluando recetarios.

La estrategia fue educativa pues la evidencia nos dice que, si se mejoran las prácticas en la alimentación, la nutrición complementaria y el cuidado en los niños, la recuperación de la anemia es sostenible. Esta estrategia fue desarrollada por medio de capacitaciones teniendo como ejes fundamentales tres contenidos básicos: (a) Alimentación complementaria. (b) Importancia de la anemia y consumo de micronutrientes y c) Lavado de manos e higiene de alimentos (por la asociación existente con diarreas frecuentes). Las promotoras comunales, con los conocimientos adquiridos en las capacitaciones, son las responsables de realizar los acompañamientos domiciliarios para mejorar hábitos de alimentación, además de retroalimentar la intervención integral en sesiones semanales. Adicionalmente, se encargaron de desarrollar con las mamás o cuidadores/as, en los hogares, nuevos recetarios ricos en hierro, en función de sus ingresos y oferta local. Si analizamos la figura No. 1, debe ponerse énfasis en que las madres o cuidadores/as, son los actores de mayor importancia por su relación permanente y directa con los niños, encargadas de poner en práctica el mejoramiento de los hábitos alimenticios a nivel del hogar, además de retroalimentar la intervención en sesiones semanales. 
FIGURA N 1.- Flor de Amancaes: Esquema del diseño de la intervención educativa

Enfoque metodológico

Proceso de enseñanzaaprendizaje en adultos, rescatando saberes previos y roles, desarrollando capacidades y competencias en promotoras comunales, madres y cuidadores/as de niños y niñas con anemia, para mejora de hábitos de alimentación complementaria.
Desarrollo de Capacidades y Competencias

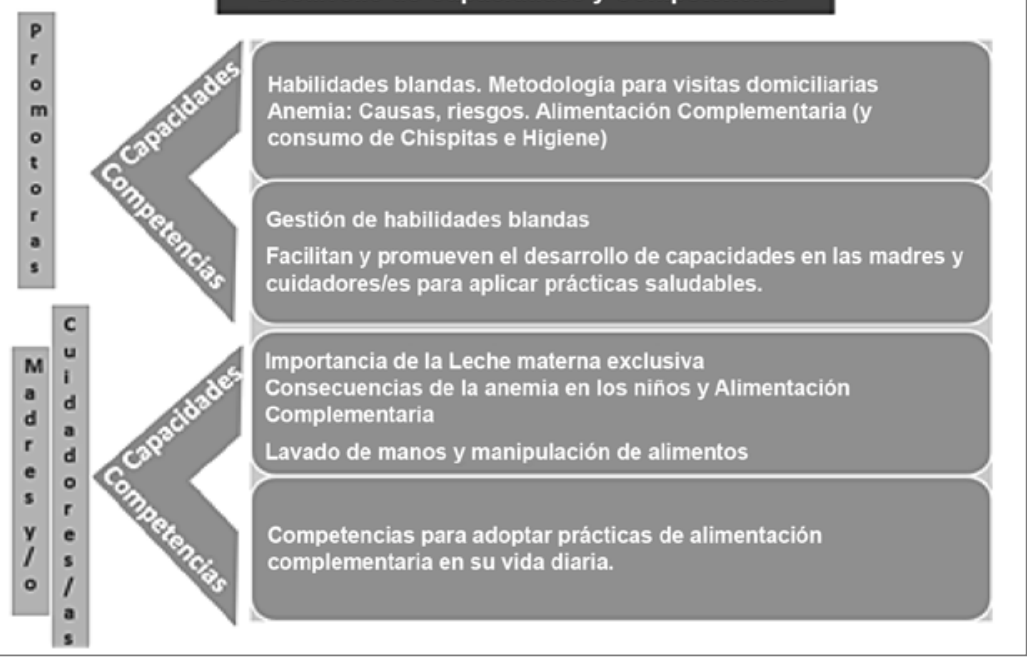

Fuente y elaboración: Propia.

La metodología de intervención en las madres de familia diseñada previamente y en contacto con la realidad- se efectivizó a través de una intervención educativa de acompañamiento y consejería, que tuvo un enfoque metodológico previo en desarrollar las capacidades y competencias en las promotoras comunales. La figura $\mathrm{N}^{\circ} 2$, muestra el diseño muestral de la población de los hogares del asentamiento humano Flor de Amancaes, donde se eligió una muestra científica de 400 hogares para obtener resultados con un nivel de confianza del 95\%.
El diseño de la muestra fue probabilístico. En la primera etapa, se observa cómo la población residente era relativamente homogénea al pertenecer a los estratos de menores ingresos seleccionando algunos sectores. En la segunda etapa, se hizo la selección de las viviendas, brindado la capacitación a través de un programa estructurado.

\section{FIGURA N$^{\circ}$ 2.- Diseño muestral en asentamiento humano Flor de Amancaes}

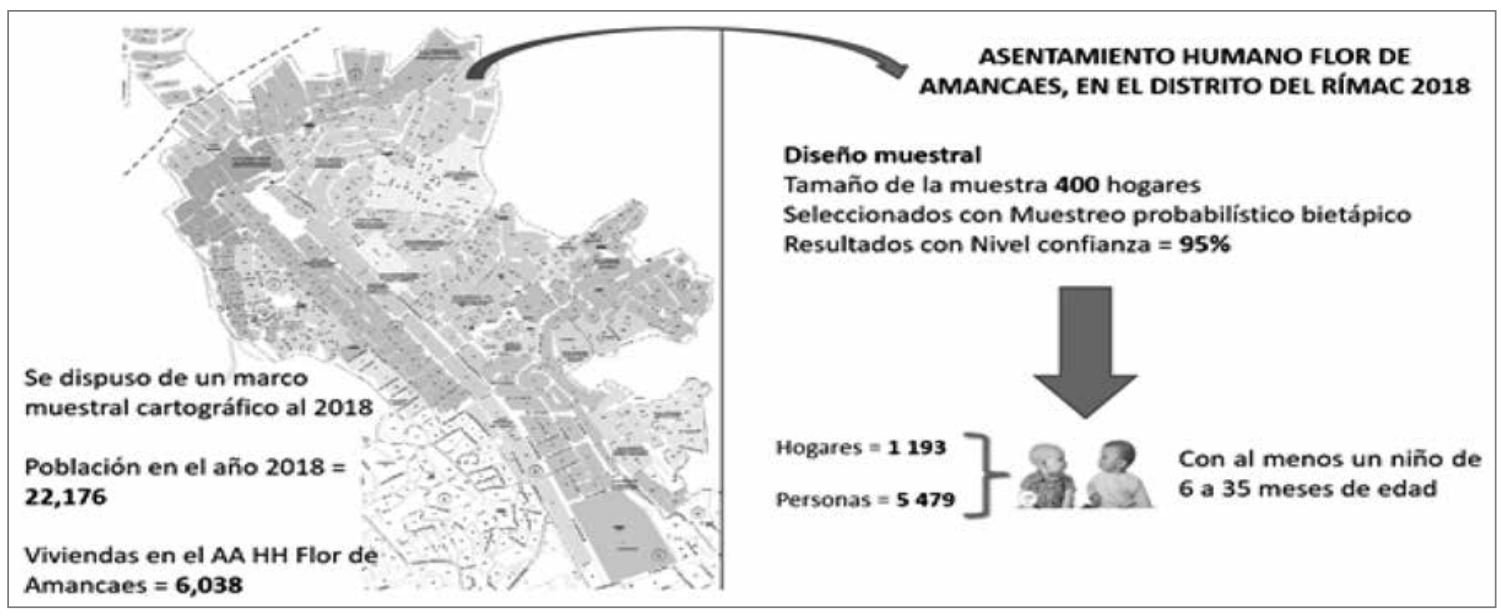

Fuente y elaboración: Propia. 
entre 6 y 36 meses: el caso del área urbano marginal de Flor de Amancaes, Lima, Perú

Importance of educational intervention as a preventive factor of anemia in children between

6 and 36 months old: the case of inner-city areas of Flor de Amancaes, Lima, Peru

Al evaluar la figura $\mathrm{N}^{\circ} 3$ que muestra los protocolos de acompañamiento domiciliados las promotoras a través de visitas, explicaron a las madres o cuidadores/as los siguientes conocimientos: (a) Importancia de la anemia y consumo de micronutrientes, (b) Lavado de manos e higiene de alimentos, por la asociación existente con diarreas frecuentes, (c) Contenidos conceptuales sobre anemia y consumo de micronutrientes; preparaciones a ser recomendadas y absolución de dudas y/o consultas realizadas por las madres y/o cuidadoras/es de las niñas y niños con anemia, en las visitas domiciliarias y (d) Recomendaciones sobre combinación adecuada de alimentos e higiene.

\section{FIGURA No 3.- Protocolo del acompañamiento con intervención educativa}

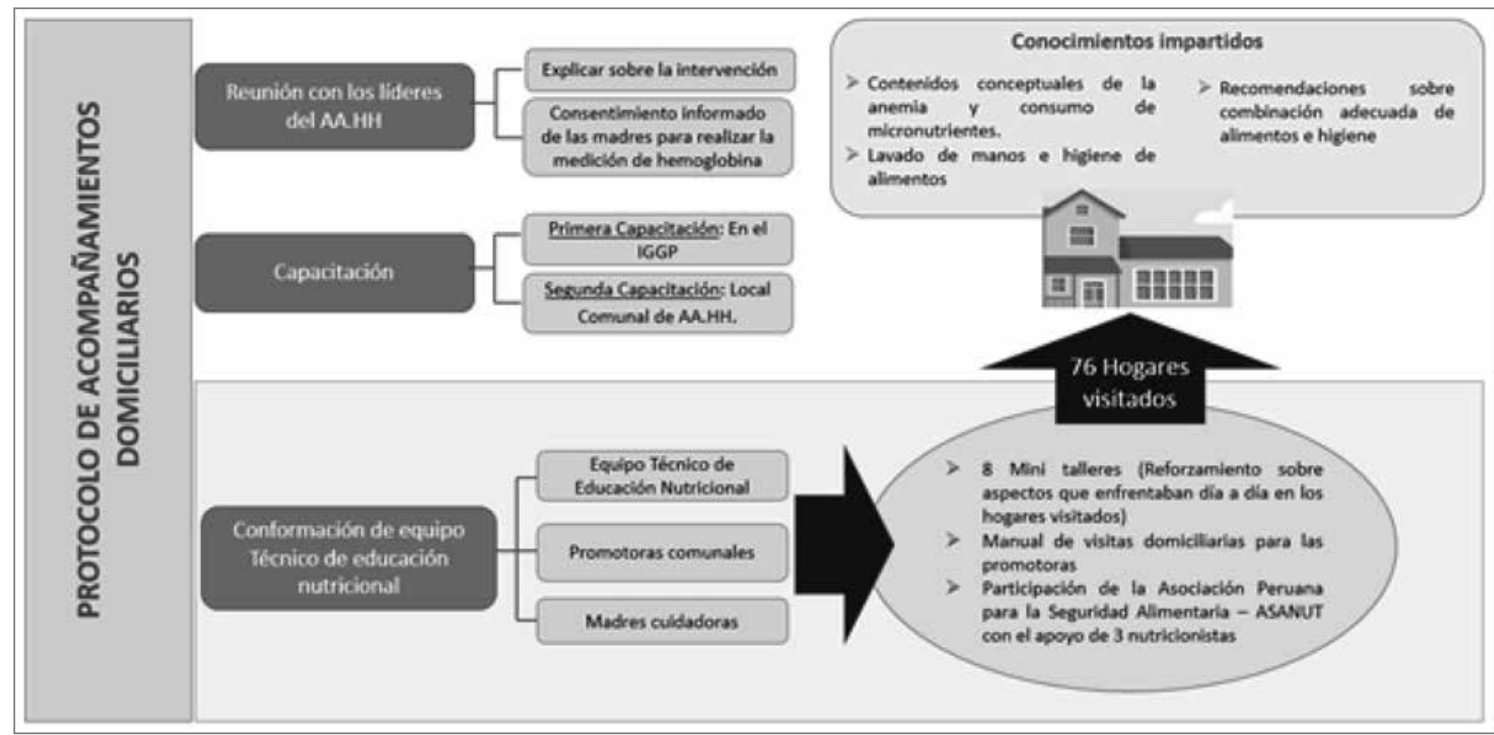

Fuente y elaboración: Propia.

Como una de las primeras acciones realizadas para el buen desarrollo de la intervención, se tuvo una reunión con las líderes del asentamiento humano, con la finalidad de explicar que la intervención era para reducir la anemia en los niños de la zona, además de que iba a ser necesario el consentimiento informado de las madres para realizar la medición de hemoglobina. Este trabajo de capacitación fue realizado en dos talleres previos a la intervención en campo.

Se realizaron dos talleres, el primero se llevó a cabo en el mes de octubre del 2018 en las instalaciones del Instituto de Gobierno y Gestión Pública de la USMP, haciendo uso para ello de un Manual de Alimentación Complementaria y de una Guía de Visitas Domiciliarias. La segunda capacitación se efectuó en el local comunal del asentamiento humano Flor de Amancaes, ese mismo año. Adicionalmente se desarrollaron ocho minitalleres -en la propia zona de trabajo- en los que se reforzaron aspectos concretos que ellas enfrentaban en su relación día a día en los hogares que visitaban.

Asimismo, se hizo entrega a las promotoras de los materiales de consulta para recordar conceptos o reforzar mensajes de forma rápida y segura. El Manual de Visitas Domiciliarias -que también fue elaborado ad hoc-pretendió recordar aspectos que aseguren una visita domiciliaria exitosa, básicamente en lo relacionado al abordaje sensible, respetuoso, persuasivo y tolerante. Para ello, se contó con la activa participación de la Asociación Peruana para la Seguridad Alimentaria ASANUT, con el aporte de tres nutricionistas quienes realizaron visitas de campo todos 
los sábados, desarrollando el reforzamiento de conocimientos y capacidades en los mini-talleres y las visitas domiciliarias a las madres de niñas y niños con anemia. En todo el periodo de la intervención, se realizaron visitas domiciliarias a 76 hogares, las cuales se ejecutaron de manera continua, a excepción de las semanas previas a la Navidad y la primera semana del mes de enero. La intervención contó con monitoreo permanente.

Dentro de las principales consejerías brindadas sobre alimentación complementaria, en función de la oferta local y de sus ingresos, se elaboró un recetario de distintas preparaciones de comida, para que las madres puedan contar con alternativas de alimentación complementaria para sus hijos en base a: bazo, hígado, sangrecita, entre otros, con alto contenido de hierro. Como lecciones aprendidas de la intervención realizada, se pueden mencionar las siguientes: (a) La estrategia de las visitas domiciliarias es la mejor intervención para mejorar las prácticas inadecuadas de cuidado y alimentación infantil. (b) La estrategia de implementación a través de la participación activa de mujeres líderes de la propia comunidad o entorno. (c) Es necesario que el establecimiento de salud tenga un rol activo. (d) Se puede afirmar que las madres principalmente las jóvenes entre los 18 a 25 años, carecen de los conocimientos necesarios para poder enfrentar con éxito la etapa de la alimentación complementaria infantil. Por lo tanto, existe un desconocimiento en la población urbano marginal en las diferentes regiones del Perú sobre el uso de los micronutrientes (chispitas) y las formas de consumo de alimentos balanceados, ricos en hierro, de bajo costo y accesibles de manera regular lo que permitiría la reducción de la anemia en nuestro país. Por lo que se debe implementar esta intervención a través de la participación activa de mujeres líderes de la propia comunidad y otras adyacentes.

\section{CONCLUSIONES}

La dación de normas como formulación de planes y elaboración de guías técnicas para luchar contra la anemia en niños y adolescentes, sirve para que tengamos claro que el diseño de una política pública precisa de mayores decisiones que, superan el ámbito de la regulación legal y, muestran la necesidad de crear compromisos de intervención concretos, donde la implementación de la política pública debe cuidar llegar al nivel primario de la misma, como es acompañar al núcleo familiar del menor $\mathrm{o}$ adolescente.

La ejecución de toda política pública de lucha contra la anemia, es preciso que se encuentre contenida en norma de rango legal, para asegurar su implementación, pero debe cuidar la atención directa a la familia que alberga al menor, a efectos de garantizar se haga efectiva la alimentación suplementaria.

El Estado peruano, debe incorporar en el Plan Nacional de Derechos Humanos 2018-2021, una estrategia que permita el seguimiento y mejora del indicador de anemia en niños, niñas y adolescentes, en edad escolar y no solo en la primera etapa de vida hasta los 36 meses como se plantea en la actualidad.

Finalmente deben establecerse un mecanismo de seguimiento a las madres de las niñas y niños que tuvieron $\mathrm{y} / \mathrm{o}$ tienen anemia, a fin de poder continuar brindándoles consejería y acompañamiento en educación, nutrición e investigación.

\section{FUENTES DE INFORMACIÓN}

\section{Fuentes bibliográficas}

Ministerio de Desarrollo e Inclusión Social (2018). Plan Multisectorial de Lucha contra la Anemia. Comisión Intersectorial de Asuntos Sociales-CIAS, aprobado mediante Decreto Supremo No. 68-2018-PCM del 3 de julio de 2019. Lima, Perú.

Ministerio de Salud (2015). Guía Técnica de Práctica Clínica para el Diagnóstico y Tratamiento de la Anemia por Deficiencia de Hierro en Niñas, Niños y Adolescentes en Establecimientos de Salud del Primer Nivel de Atención.

Ministerio de Salud del Perú (2017). Plan Nacional para la reducción y control de la anemia materno infantil y la desnutrición crónica infantil en el Perú: 2017 - 2021. Documento Técnico. ISBN: Biblioteca Nacional del Perú No. 2017 - 1ra. Edición. RM. No. 249-2017/MINSA. 
entre 6 y 36 meses: el caso del área urbano marginal de Flor de Amancaes, Lima, Perú

Importance of educational intervention as a preventive factor of anemia in children between

6 and 36 months old: the case of inner-city areas of Flor de Amancaes, Lima, Peru

\section{Fuentes Electrónicas.}

Aparco, JP; Bullón, L. \& Cusirramos, S. (2019). Impacto de micronutrientes en polvo sobre la anemia en niños de 10 a 35 meses de edad en Apurímac, Perú. Revista Peruana de Medicina Experimental y Salud Publica, 36(1), 17-25. Recuperado del sitio de internet: https:// dx.doi.org/10.17843/rpmesp.2019.361.4042

Arroyo-Laguna J. (2017). Hacia un Perú sin anemia. Revista Peruana de Medicina Experimentaly Salud Publica. 2017; 34(4):5867. Recuperado del sitio de internet: https://doi. org/10.17843/rpmesp.2017.344.3279

Balajaran, Y; Ramakrishnan, U; Ozaltin, E; Shankar, AH \& Subramanian SV. (2011). Anaemia in low-income and middle-income countries. The Lancet Journal. 2011; Recuperado del sitio de internet: https://doi. org/10.1016/S0140-6736(10)62304-5.

Defensoría del Pueblo (2019). Intervención del Estado para la reducción de la anemia infantil: Resultados de la supervisión nacional. Recuperado del sitio de internet: https://www.defensoria.gob.pe/wpcontent/uploads/2019/01/Informe-deAdjunt\%C3\%ADa-012-2018-DPAAE.pdf

Feldman, L; Najle, R; Rivero, F; Rodríguez, E; Estein, S. (2017) Anemia inflamatoria: fisiopatología, diagnóstico y tratamiento. Acta Bioquímica Clínica Latinoamericana, vol. 51, núm. 3, 2017, pp. 361-374Federación Bioquímica de la Provincia de Buenos AiresBuenos Aires, Argentina. Recuperado del sitio de internet: http://www.redalyc.org/ pdf/535/53553013011.pdf

Gonzales, E; Huamán-Espino, L; Gutiérrez, C; Aparco, J; Pillaca, J. (2015). Caracterización de la anemia en niños menores de cinco años de zonas urbanas de Huancavelica y Ucayali en el Perú. Revista Peruana de Medicina Experimental y Salud Publica. vol.32 no.3 Lima jul./set. 2015. ISSN 17264634. Recuperado del sitio de internet: http:// www.scielo.org.pe/scielo.php? script $=$ sci arttext\&pid $=$ S1726-46342015000300004

Hernández-Martínez， A. (2018). Anemia de Fanconi. Med Int Méx.2018 septiembreoctubre; 34 (5):730-734. Recuperado del sitio de internet: http://www.scielo.org.mx/pdf/ $\mathrm{mim} / \mathrm{v} 34 \mathrm{n} 5 / 0186-4866-\mathrm{mim}-34-05-730 . p d f$
Huamán-Espino, L; Aparco, JP; NúñezRobles, E; Gonzáles, E; Pillaca, J. \& MaytaTristán, P. (2012). Consumo de suplementos con multimicronutrientes Chispitas ${ }^{\circledR}$ y anemia en niños de 6 a 35 meses: estudio transversal en el contexto de una intervención poblacional en Apurímac, Perú. Revista Peruana de Medicina Experimental y Salud Publica, 29(3), 314323. Recuperado del sitio de internet: http:// www.scielo.org.pe/scielo.php? script $=$ sci arttext\&pid $=$ S1726-46342012000300004\&ln $\mathrm{g}=\mathrm{es} \&$ tlng $=\mathrm{es}$

INEI. (2017). Indicadores de resultados de los programas presupuestales 2012-2017. Encuesta Demográfica y de Salud Familiar 2017. Lima, febrero. Recuperado del sitio de internet: https://proyectos.inei.gob.pe/endes/images/ Peru_Indicadores_de_PPR_2012_2017.pdf

Junco, J.E. (2015). Identificación de los factores que contribuyen y limitan la efectividad del programa de suplementación con multimicronutrientes en la reducción de la anemia de niños menores de tres años del ámbito rural de Vinchos de Ayacucho [Tesis para optar el grado de magister en gerencia social]. Lima, Perú: Pontificia Universidad Católica del Perú, 2015. Recuperado del sitio de internet: http://tesis.pucp.edu.pe/repositorio/bitstream/ $\mathrm{h}$ andle/20.500.12404/6650/JUNCO GUILLERMO JORGE IDENTIFICACION ${ }_{-}^{-}$ FACTORES.pdf? sequence $=1 \&$ isAllowed $=y$

Kibret. KT; Chojenta, C; D'Arcy E, et al (2019) Spatial distribution and determinant factors of anaemia among women of reproductive age in Ethiopia: a multilevel and spatial analysis BMJ Open 2019;9:e27276. Recuperado del sitio de internet: https://doi.org/10.1136/ bmjopen-2018-027276

Martínez, LM., Rodríguez, MA., Agudelo, CA., Vargas, N., y Peña, GA. (2014). La anemia como signo de malnutrición en niños de un programa de recuperación nutricional domiciliaria en Antioquia, Colombia. Rev Univ. salud. 2014; 16(1): 105 - 113. Recuperado del sitio de internet: https://www.researchgate. net/profile/Lina_Martinez-Sanchez/ publication/317503486_Anemia_as_a_sign_ of_malnutrition_in_children_belonging to_the_home_nutritional_recovery_ program in Antioquia_Colombia/

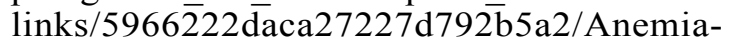
as-a-sign-of-malnutrition-in-childrenbelonging-to-the-home-nutritional-recoveryprogram-in-Antioquia-Colombia.pdf 
Ministerio de Justicia y Derechos Humanos (s/f). Plan Nacional de Derechos Humanos 2018-2021. Recuperado del sitio de internet: http://spij.minjus.gob.pe/content/banner secundario/img/muestra/PLAN-ANUAL.pdf

Naciones Unidas (2015). Memoria del Secretario General sobre la labor de la Organización. Recuperado del sitio de internet: https://undocs.org/es/A/70/1

Organización Mundial de la Salud (2011) concentraciones de hemoglobina para diagnosticar la anemia y evaluar su gravedad. Ginebra. 2011. Recuperado del sitio de internet: https://www.who.int/vmnis/indicators/ haemoglobin_es.pdf

Organización Mundial de la Salud (2017). Salud y derechos humanos. Recuperado del sitio de internet: https://www.who.int/es/newsroom/fact-sheets/detail/human-rights-andhealth

Pita-Rodríguez, Gisela, \& Jiménez-Acosta, Santa. (2011). La anemia por deficiencia de hierro en la población infantil de Cuba. Brechas por cerrar. Revista Cubana de Hematología, Inmunología y Hemoterapia, 27(2), 179195. Recuperado del sitio de internet: http://scielo.sld.cu/scielo.php? script $=$ sci arttext\&pid=S0864-02892011000200003\&lng $=$ es\&tlng $=$ es.
Zabaleta, N. \& Astete, L (2017), Efecto de la anemia en el desarrollo infantil: consecuencias a largo plazo. Revista Peruana de Medicina Experimental y Salud Publica. vol.34 no.4 Lima oct./dic. 2017. ISSN 1726-4634. Recuperado del sitio de internet: http://dx.doi. org/10.17843/rpmesp.2017.344.3346

\section{Fuentes normativas}

\section{Nacional}

Congreso de la República (1997). Ley $\mathrm{N}^{\circ}$ 26842. Ley General de Salud. En Diario oficial El Peruano. Lima, Perú.

\section{Internacional}

Asamblea General de las Naciones Unidas (1948). La Declaración Universal de Derechos Humanos. 